\title{
Evaluation of Diaphragmatic Ultrasound Indices as Predictors of Successful Liberation From Mechanical Ventilation in Subjects With Abdominal Sepsis
}

\author{
Haytham H Eltrabili, Ahmed M Hasanin, Mahmoud S Soliman, Ahmed M Lotfy, \\ Walid I Hamimy, and Ahmed M Mukhtar
}

\begin{abstract}
BACKGROUND: Sepsis-induced diaphragmatic dysfunction is one of the main risk factors of failure to liberate patients from mechanical ventilation. Several studies addressed diaphragmatic ultrasound as a valuable tool in the assessment of diaphragmatic function during liberation from mechanical ventilation in different populations. However, none of these studies examined the use of diaphragmatic ultrasound to predict failure of liberation from mechanical ventilation in subjects with sepsis METHODS: A prospective observational study was done with subjects on mechanical ventilation and with abdominal sepsis. The diaphragmatic thickening fraction, diaphragmatic excursion, and rapid shallow breathing index were assessed $30 \mathrm{~min}$ after a spontaneous breathing trial RESULTS: Thirty subjects were enrolled in the study. Seventeen subjects were successfully extubated $(56.6 \%)$, whereas extubation failed in 13 subjects $(43.4 \%)$. The time to the first liberation attempt was significantly shorter in the liberation-success group $2.3(0.7) \mathrm{d}$ compared with the liberation-failure group $5.8(4.7) \mathrm{d} ; \boldsymbol{P}=.02$. The optimum cutoff value of diaphragmatic thickening fraction for predicting liberation success was $\geq 30.7 \%$, with a sensitivity of $94.1 \%$ and a specificity of $100 \%$. The area under the curve was 0.977 . Although diaphragmatic excursion of $\geq 10.4 \mathrm{~mm}$ had a sensitivity of $94 \%$ and a specificity of $85 \%$ for predicting liberation success, with an area under the curve of 0.85 . A rapid shallow breathing index of $\leq 44$ had a specificity of $100 \%$ and a sensitivity of $76 \%$; the area under the curve was 0.9. CONCLUSIONS: Diaphragmatic ultrasound indices, namely diaphragmatic thickening fraction and diaphragmatic excursion, could be useful parameters for assessment of success of liberation in patients on mechanical ventilation with abdominal sepsis. (ClinicalTrials.gov registration NCT03094299.) Key words: abdominal sepsis; delayed extubation; diaphragmatic thickening fraction; liberation failure; mechanical ventilation; premature liberation. [Respir Care 2019;64(5):564-569. @ 2019 Daedalus Enterprises]
\end{abstract}

\section{Introduction}

The optimum time for extubation of patients who are critically ill and on mechanical ventilation remains one of

Drs Eltrabili, Hasanin, Soliman, Lotfy, Hamimy, and Mukhtar are affiliation with the Department of Anesthesia and Critical Care, Cairo University Hospital, Cairo, Egypt.

This work was funded by Cairo University.

The authors have disclosed no conflicts of interest.

Presented the abstract of this article as an oral presentation at ESICM Lives, held September 2017, in Vienna, Austria. the most-challenging decisions in intensive care. Intensivists should maintain the balance between the risk of premature liberation from mechanical ventilation with morbidities of extubation failure and the risk of delayed extubation, which increases the duration of mechanical ventilation. Current guidelines recommend several indices to predict liberation success during spontaneous breathing

\footnotetext{
Correspondence: Ahmed Hasanin MD, Department of Anesthesia and Critical Care Medicine, 01 Elsarayah Street, Elmanyal, Cairo 11559, Egypt. E-mail: ahmedmohamedhasanin@gmail.com.
}

DOI: $10.4187 /$ respcare.06391 
trials (SBTs), ${ }^{1}$ although none have proven to be ideal. ${ }^{2}$ The reason for this is likely because of the heterogeneity of patients who are critically ill, which limits the predictive abilities of these indices in different subgroups of patients. Individuals with sepsis are a subgroup of patients who are critically ill and who are considered to be at high risk of liberation failure. ${ }^{3}$ Although the mechanism remains poorly understood, sepsis-induced diaphragmatic dysfunction is one of the leading causes of liberation failure in these patients. $^{4}$

Currently, there is great interest in assessing diaphragmatic function during an SBT by ultrasound to predict liberation failure. A recent meta-analysis reported the results of 19 studies that assessed diaphragmatic function by using ultrasound with different values of sensitivity and specificity. ${ }^{5}$ Most of these studies included subjects from different populations; however, none examined the use of diaphragmatic ultrasound to predict liberation failure in subjects with sepsis. We sought to evaluate the efficacy of ultrasound-derived variables in the prediction of success of liberation from mechanical ventilation in patients who are critically ill and with abdominal sepsis.

\section{Methods}

This prospective observational study was carried out in a surgical ICU unit of a trauma and emergency department at Cairo University teaching hospital over a 12-month period. The study was approved by the research ethics committee (N-16-2017) and was registered at ClinicalTrials. gov before subject enrollment (NCT03094299). Informed consent was obtained from each subject's next of kin before commencement of the study. All the subjects on mechanical ventilation for $>48 \mathrm{~h}$ and with intra-abdominal sepsis were consecutively included. A sepsis diagnosis was established once the criteria of the American College of Chest Physicians/Society of Critical Care Medicine Consensus Conference ${ }^{6}$ were met. A diagnosis of abdominal sepsis was confirmed by abdominal imaging (computed tomography or ultrasound) or during surgical exploration. The exclusion criteria were as follows: patients $<18$ y old; those with diaphragmatic paralysis, surgery, injury, or a surgical dressing over the right lower rib cage that would preclude ultrasound examination; and patients with neuromuscular disease.

\section{Assessment of Readiness to Liberate From Mechanical Ventilation}

Assessment of readiness to liberate patients from mechanical ventilation was done by an ICU physician according to our protocol, which included the following: (a) resolution of an acute phase of disease for which the subject

\section{QUICK LOOK}

\section{Current knowledge}

Sepsis-induced diaphragmatic dysfunction is a major risk factor of weaning failure during mechanical ventilation. Diaphragmatic ultrasound had been a useful tool for predicting successful liberation from mechanical ventilation.

\section{What this paper contributes to our knowledge}

Using ultrasound, we evaluated diaphragmatic movement at the eighth or ninth intercostal space, between the anterior and the mid axillary lines on the right side to observe the zone of apposition of the muscle 0.5 to $2 \mathrm{~cm}$ below the costophrenic sinus. We found that diaphragmatic excursion and diaphragmatic thickening fraction showed a good predictive ability for successful liberation from mechanical ventilation in subjects with abdominal sepsis.

was intubated; (b) adequate cough and absence of excessive tracheobronchial secretion; (c) adequate oxygenation, $\mathrm{P}_{\mathrm{aO}_{2}}>60 \mathrm{~mm} \mathrm{Hg}$, with PEEP $\leq 8 \mathrm{~cm} \mathrm{H} \mathrm{H}_{2} \mathrm{O}$, and $\mathrm{F}_{\mathrm{IO}_{2}} \leq 0.5$; (d) breathing frequency $\leq 30$ breaths $/ \mathrm{min}$, $\mathrm{pH}$, and $\mathrm{P}_{\mathrm{aCO}_{2}}$ appropriate for the subject's baseline respiratory status; (e) stable cardiovascular status (ie, heart rate $<120$ beats/min; systolic blood pressure, $90-$ $160 \mathrm{~mm} \mathrm{Hg}$; and minimum or no vasopressor use, such as dopamine or dobutamine $<5 \mu \mathrm{g} / \mathrm{kg} / \mathrm{min}$ or noradrenaline $<0.05 \mu \mathrm{g} / \mathrm{kg} / \mathrm{min}$ ).

\section{SBT}

Subjects scheduled for an SBT were put on pressure support mode with a pressure support of $5 \mathrm{~cm} \mathrm{H}_{2} \mathrm{O}$ and PEEP of $5 \mathrm{~cm} \mathrm{H}_{2} \mathrm{O}$ for $30 \mathrm{~min}$, then all liberation parameters were again assessed. The decision to extubate was made by the intensivist in charge who was blinded to the ultrasonographic measurements. Liberation failure was defined as either re-intubation or use of noninvasive ventilation within $48 \mathrm{~h}$ of extubation.

\section{Ultrasound Assessment for the Diaphragm}

All the subjects were evaluated while they were in a semi-recumbent position by using a Mindray model M7 ultrasound machine with a 7.5-10 MHz linear and 3-5 curvilinear probes (Mindray Bio-Medical, Shenzhen, China) set to the B mode. 


\section{Diaphragmatic Thickening Fraction}

The linear probe was placed perpendicular to the chest wall, in the eighth or ninth intercostal space, between the anterior and the mid axillary lines on the right side to observe the zone of apposition of the muscle 0.5 to $2 \mathrm{~cm}$ below the costophrenic sinus. The diaphragm was imaged as a structure with 3 distinct layers, including 2 parallel echoic lines (the diaphragmatic pleura and the peritoneal membrane) and a hypoechoic structure between them (the muscle itself). The diaphragm thickness was measured on the right side from the middle of the pleural line to the middle of the peritoneal line after obtaining $\mathrm{M}$-mode tracing of the contracting diaphragm. Three measurements were taken over 3 respiratory cycles and were averaged. Diaphragmatic thickening fractions were calculated as follow: (thickness at end inspiration - thickness at end expiration)/thickness at end expiration.

\section{Diaphragmatic Excursion}

The curvilinear probe was placed over one of the lower intercostal spaces in the right anterior axillary line for the right diaphragm and the left mid axillary line for the left diaphragm. With the probe fixed on the chest wall during respiration, the ultrasound beam was directed to the hemidiaphragmatic domes at an angle of not $<70^{\circ}$. During inspiration, the normal diaphragm contracts and moves caudally toward the transducer; this was recorded as an upward motion of the M-mode tracing. The amplitude of excursion was measured on the vertical axis of the tracing from the baseline to the point of maximum height of inspiration.

\section{Outcomes}

Primary Outcome. The primary outcome was the area under the receiver operating characteristic curve for ultrasonographic measurements of the diaphragm (diaphragmatic thickening fraction, and diaphragmatic excursion) to predict liberation failure.

Secondary Outcomes. Secondary outcomes were hemodynamic, ventilatory, and ultrasonographic data: heart rate, systolic blood pressure, and breathing frequency were recorded at baseline (before SBT) and $30 \mathrm{~min}$ thereafter.

Diaphragmatic muscle thickness during inspiration, during expiration, diaphragmatic thickening fraction, diaphragmatic excursion, and rapid shallow breathing index (RSBI) were recorded $30 \mathrm{~min}$ after SBT. RSBI was calculated by dividing breathing frequency/tidal volume. Other data, which included demographic information, severity scores, $\mathrm{P}_{\mathrm{aO}} / \mathrm{F}_{\mathrm{IO}_{2}}$, duration of mechanical ventilation (d), ICU length of stay, and hospital mortality, were collected.

\section{Statistical Analysis and Sample Size Calculation}

We calculated our sample size by using MedCalc version 12.1.4.0 (MedCalc Software bvba, Mariakerke, Belgium) to detect the area under the receiver operating characteristic curve of at least 0.8 for ultrasonographic measurements of the diaphragm to predict liberation failure, with a null hypothesis of the area under the receiver operating characteristic curve of 0.5 . The minimum number of the subjects needed to have a study power of $80 \%$ and alpha error of 0.05 was 26 , with at least 13 positive (weaning failure) subjects.

Descriptive statistics were presented as mean \pm SD or as median and interquartile range for continuous variables, and as absolute or relative frequencies for categorical variables. The unpaired Student $t$ test or Mann-Whitney test were used to compare continuous variables and chi-square tests, or Fisher exact tests were used to compare categorical variables. To compare the performance of diaphragmatic thickening fraction, diaphragmatic excursion, and RSBI in predicting liberation failure, receiver operating characteristic curves were constructed and the area under the curve was calculated. MedCalc generated values with the highest sensitivity and specificity (Youden index). The areas under the receiver operating characteristic curves were compared by using the Hanley-McNeil test. The level of significance was set at $P<.05$ for 2-tailed tests.

\section{Results}

Forty patients were screened, 35 patients met the inclusion criteria, and 30 subjects were available for final analysis. The reason for exclusion was surgery over the right lower thoracic cage. The most-frequent causes of abdominal sepsis were perforated viscous (15 subjects), intestinal obstruction (10 subjects), and mesenteric vascular occlusion (5 subjects). All the subjects were admitted to ICU after abdominal exploration and were intubated and on mechanical ventilation. Seventeen subjects $(56.6 \%)$ were successfully extubated; whereas extubation failed in 13 subjects $(43.4 \%)$. The subjects in the liberation-success group had a significantly lower APACHE II (Acute Physiology and Chronic Health Evaluation II) score and SOFA (Sequential Organ Failure Assessment) score compared with those in the liberation-failure group. Other demographic and clinical data did not differ between the groups (Table 1). The length of ICU stay was significantly shorter in the subjects who were successfully liberated than in those for whom the liberation attempt failed. The overall survival was $42 \%$. Survival in the liberation-success group was significantly higher than the liberation-failure group, 50\% and $30 \%$, respectively $(P=0.006)$. 


\section{Diaphragmatic Ultrasound to Predict Weaning in Sepsis}

Table 1. Demographic Data and Subject Characteristics

\begin{tabular}{|c|c|c|c|}
\hline Characteristic & Liberation Success $(n=17)$ & Liberation Failure $(n=13)$ & $P$ \\
\hline Age, mean $\pm \mathrm{SD}, \mathrm{y}$ & $52.7 \pm 13.4$ & $51.4 \pm 13.1$ & .79 \\
\hline Males, $n(\%)$ & $11(65)$ & $5(39)$ & .27 \\
\hline APACHE II score, mean \pm SD & $22.7 \pm 1.9$ & $25 \pm 1.8$ & .002 \\
\hline SOFA score, mean $\pm \mathrm{SD}$ & $5.1 \pm 1.1$ & $8.3 \pm 1.2$ & $<.001$ \\
\hline Time to first weaning attempt, mean \pm SD & $2.3 \pm 0.7^{*}$ & $5.8 \pm 4.7$ & .001 \\
\hline Postoperative days, median (quartiles) & $2(2-3)^{*}$ & $3(2.5-9)$ & .007 \\
\hline Survival, $n(\%)$ & $9(53)^{*}$ & $4(31)$ & .006 \\
\hline Time to death, median (quartiles) d & $12(6-17)$ & $8(9-16)$ & .62 \\
\hline Cause of death, $n(\%)$ & & & $>.99$ \\
\hline Sepsis & $7(87)$ & $8(89)$ & \\
\hline Cardiogenic shock & $1(13)$ & $1(11)$ & \\
\hline \multicolumn{4}{|c|}{$\begin{array}{l}\text { * Denotes statistical significance. } \\
\text { APACHE II = Acute Physiology and Chronic Health Evaluation II } \\
\text { SOFA = Sequential Organ Failure assessment } \\
\text { IQR = interquartile range }\end{array}$} \\
\hline
\end{tabular}

Table 2. Hemodynamic, Ventilator, and Ultrasonographic Data

\begin{tabular}{|c|c|c|c|}
\hline Parameter & Liberation Success $(n=17)$ & Liberation Failure $(n=13)$ & $P$ \\
\hline \multicolumn{4}{|l|}{ Heart rate mean $(\mathrm{SD})$, beats/min } \\
\hline Baseline & $90(15)$ & $98(13)$ & .17 \\
\hline After $30 \mathrm{~min}$ of SBT & $92(10)$ & $98(21)$ & .30 \\
\hline \multicolumn{4}{|l|}{ SBP mean $(\mathrm{SD}), \mathrm{mm} \mathrm{Hg}$} \\
\hline Baseline & $122(15)$ & $132(15)$ & .08 \\
\hline After $30 \mathrm{~min}$ of SBT & $120(14)$ & $123(11)$ & .53 \\
\hline \multicolumn{4}{|l|}{ Breathing frequency mean (SD), breaths/min } \\
\hline Baseline & $19(3.4)$ & $21.3(3)$ & .06 \\
\hline After $30 \mathrm{~min}$ of SBT & $18(4)^{*}$ & $27(4)$ & $<.001$ \\
\hline RSBI mean (SD) & $39(18)^{*}$ & $77(30)$ & $<.001$ \\
\hline Inspiratory thickness, median (quartiles) $\mathrm{mm}$ & $2.7(2.4-3)$ & $2.2(1.9-3.4)$ & .30 \\
\hline Expiratory thickness, median (quartiles) mm & $2(1.65-2.1)$ & $2(1.6-2.6)$ & .62 \\
\hline DTF, median (quartiles) $\%$ & $42.1(39.4-48.5)^{*}$ & $21.4(14.6-30.7)$ & .007 \\
\hline $\mathrm{DE}$, median (quartiles) $\mathrm{mm}$ & $17(14.4-23.4)^{*}$ & $8(5.2-10.2)$ & $<.001$ \\
\hline $\begin{array}{l}\text { *Denotes statistical significance. } \\
\text { SBT = spontaneous breathing trial } \\
\text { SBP = systolic blood pressure } \\
\text { RSBI = rapid shallow breathing index } \\
\text { IQR = interquartile range } \\
\text { DTF = diaphragmatic thickening fraction } \\
\text { DE = diaphragmatic excursion }\end{array}$ & & & \\
\hline
\end{tabular}

The time to the first liberation attempt was significantly lower in the liberation-success group, $2.3(0.7) \mathrm{d}$, compared with the liberation-failure group, $5.8(4.7) \mathrm{d} ; P=.02$. Both heart rate and systolic blood pressure were comparable between the groups before and $30 \mathrm{~min}$ after the SBT (Table 2). Baseline frequency did not differ between the groups; however, it increased significantly $30 \mathrm{~min}$ after the SBT (Table 2), The central venous pressure before the SBT was significantly lower in the liberation-success group, 9.3 (2) $\mathrm{cm} \mathrm{H}_{2} \mathrm{O}$, compared with the liberation-failure group, 11.7 (2.8) $\mathrm{cm} \mathrm{H}_{2} \mathrm{O} ; P=.01$.

\section{Ventilatory and Ultrasonographic Parameters as a Predictor of Weaning Failure}

Thirty minutes after the SBT, the absolute inspiratory and expiratory diaphragmatic muscle thickness did not differ between the groups. However, both diaphragmatic thickening fraction and diaphragmatic excursion differed significantly between the subjects who were successfully liberated and those for whom the liberation attempt failed. (Table 2) The cutoff value, sensitivity, specificity, and the areas under the receiver operating 


\section{Diaphragmatic Ultrasound to Predict Weaning in Sepsis}

Table 3. Accuracy of DTF, DE, and RSBI in Predicting Liberation Success

\begin{tabular}{llcrr}
\hline \hline Parameter & Cutoff Value & $\begin{array}{c}\text { Area Under Receiver Operating } \\
\text { Characteristic Curve (95\% CI) }\end{array}$ & \% Sensitivity & $\%$ Specificity \\
\hline DTF & $\geq 30.7 \%$ & $0.98(0.93-1.0)[\mathrm{g} 3]$ & 94 & 100 \\
DE & $>10.4 \mathrm{~mm}$ & $0.85(0.74-0.97[\mathrm{~g} 6])$ & 94 & 84 \\
RSBI & $\leq 44$ & $0.9(0.7-0.98)$ & 76 & $<.001$ \\
& & & $<.001$
\end{tabular}

$\overline{\mathrm{DTF}}=$ diaphragmatic thickening fraction

$\mathrm{DE}=$ diaphragmatic excursion

RSBI $=$ rapid shallow breathing index

characteristic curves for the variables examined are shown in Table 3.

The optimum cutoff value of the diaphragmatic thickening fraction for predicting liberation success according to the maximum Youden index was $\geq 30.7 \%$. This cutoff value had a sensitivity of $94.1 \%$ and specificity of $100 \%$; the area under the curve was 0.98 (95\% CI $0.98-1$; $P<.001)$. The cutoff value of excursion that predicted liberation success was $\geq 10.4 \mathrm{~mm}$, with a sensitivity of $94 \%$ and a specificity of $84 \%$; the area under the curve was 0.85 (95\% CI $0.74-0.97 ; P<.001)$. The median and interquartile range of RSBI differed significantly between the subjects who were successfully liberated and those for whom the liberation trial failed (Table 2). Use of an RSBI cutoff value of $\leq 44$ breaths $/ \mathrm{min} / \mathrm{L}$ yielded a specificity of $100 \%$ and a sensitivity of $76 \%$; the area under the curve was 0.9 (95\% CI $0.7-0.98 ; P<.001)$ for predicting liberation success (Table 3 ).

\section{Discussion}

The main finding of the present study was that diaphragmatic parameters assessed by ultrasonography showed good ability to predict the outcome of the liberation process of adult subjects on mechanical ventilation and who were diagnosed with abdominal sepsis. To our knowledge, this study was the first to evaluate the ability of diaphragmatic ultrasound measurements to predict liberation outcome in subjects with abdominal sepsis. We found that a diaphragmatic thickening fraction of $\geq 30.7 \%$ and diaphragmatic excursion of $\geq 10.4 \mathrm{~mm}$ during SBT in the subjects with sepsis had a sensitivity and a specificity of $100 \%, 94.1 \%$ and $94 \%, 85 \%$, respectively, for predicting liberation success in these subjects.

A recent meta-analysis, which included 19 studies and involved 1,071 people, found that the pooled sensitivity for diaphragmatic excursion was $75 \%$ and pooled specificity was $75 \% .^{5}$ The high predictive values of diaphragmatic thickening fraction and diaphragmatic excursion that we found in our study might be explained by the type of population under investigation. Sepsis has been reported to be associated with diaphragmatic dysfunction. ${ }^{4}$ Several mechanisms have been implicated in the pathogenesis of sepsis-induced diaphragmatic dysfunction. One of the studies found that the incidence of nerve conduction abnormalities may reach $63 \%$ in subjects in the ICU within $72 \mathrm{~h}$ of developing sepsis. ${ }^{7}$ Diaphragmatic muscle atrophy is another mechanism of sepsis-induced diaphragmatic dysfunction. Jung et $\mathrm{al}^{8}$ found that there was a preferential loss of diaphragm muscle volume compared with peripheral muscle in subjects with sepsis. In our study, neither the absolute inspiratory nor expiratory muscle thickness differed between both groups, which dismisses the theory of diaphragmatic muscle atrophy as the most probable cause of sepsis-induced diaphragmatic dysfunction in our cohort.

Sepsis resolution and hemodynamic stability were prerequisites in our cohort's first liberation attempt, which ruled out uncontrolled sepsis as a cause of liberation failure in these subjects. One important finding of our study was that the APACHE II score was significantly lower in those successfully liberated from mechanical ventilation than in those for whom the liberation attempt failed, which may indicate that diaphragmatic dysfunction behaved as any other organ failure and may explain liberation failure in these patients. Consistent with this finding, Demoule et $\mathrm{al}^{4}$ established that the Simplified Acute Physiology Score was independently associated with diaphragm dysfunction.

In the present study, we found that the best cutoff value to predict liberation success was $\geq 30.7 \%$. The optimum cutoff value to predict liberation success is not consistent among studies and ranges from 20 to $36 \%$, depending on the ventilator support provided during the measurement; the higher the support, the lower the diaphragmatic thickening fraction. ${ }^{9-12}$ Although we did not find statistically significant differences between diaphragmatic thickening fraction and diaphragmatic excursion as indices for liberation, the area under the curve tended to be higher in the diaphragmatic thickening fraction method. In patients who have undergone abdominal surgery, abnormal respiratory movements are common. Rib cage movements during inspiration are proportionally greater, and abdominal movement is small or even paradoxical. ${ }^{13}$ Thus, measurement of 


\section{Diaphragmatic Ultrasound to Predict Weaning in SePsis}

diaphragmatic excursion may underestimate diaphragmatic function. Umbrello et $\mathrm{al}^{14}$ observed that the diaphragmatic thickening fraction is a reliable indicator of respiratory effort, whereas diaphragmatic excursion should not be used to quantitatively assess diaphragm contractile activity.

In the current study, RSBI was significantly higher in the liberation-failure group compared with the liberationsuccess group. Amoateng-Adjepong et $\mathrm{al}^{3}$ were the first to describe the effect of sepsis on the breathing pattern during a liberation trial. They found that the subjects who were recovering from sepsis had a higher RSBI compared with subjects without sepsis. ${ }^{3}$ Spadaro et al $^{15}$ found that the median (quartiles) RSBI in subjects for whom a liberation trial failed was 63 (73-90), which was more or less similar to our finding; however, the best cutoff value for predicting liberation failure in their study was $>62$, which is much higher than the threshold that was found in our study.

The first study to describe RSBI was done by Yang and Tobin ${ }^{16}$ and they found that a cutoff value $<105$ was the best threshold to predict liberation success. However, several other studies reported different thresholds for predicting liberation success with variable sensitivities and specificities, which may be due to differences in the studied population and whether the weaning method was a T-piece or pressure support. Our study had some limitations. First, the sample size was too small to draw a conclusion regarding the superiority of diaphragmatic ultrasound indices compared with RSBI. Second, we chose to only evaluate the right hemidiaphragm because the acoustic window provided by the liver made it easier to take the diaphragmatic thickening fraction measurement.

\section{Conclusions}

Both diaphragmatic ultrasound indices, namely diaphragmatic thickening fraction and diaphragmatic excursion, could be useful parameters for assessment of the success of liberation of patients on mechanical ventilation with abdominal sepsis. Further randomized controlled studies are warranted to explore the effect of incorporating diaphragmatic ultrasound in the liberation protocol on the incidence of re-intubation.

\section{REFERENCES}

1. Girard TD, Alhazzani W, Kress JP, Ouellette DR, Schmidt GA, Truwit JD, et al.; ATS/CHEST Ad Hoc Committee on Liberation from Mechanical Ventilation in Adults. An Official American Thoracic Society/American College of Chest Physicians Clinical Prac- tice Guideline: Liberation from mechanical ventilation in critically ill adults rehabilitation protocols, ventilator liberation protocols, and cuff leak tests. Am J Respir Crit Care Med 2017;195(1):120-133.

2. El-Khatib MF, Bou-Khalil P. Clinical review: liberation from mechanical ventilation. Crit Care 2008;12(4):211.

3. Amoateng-Adjepong Y, Jacob BK, Ahmad M, Manthous CA. The effect of sepsis on breathing pattern and weaning outcomes in patients recovering from respiratory failure. Chest 1997;112(2):472477.

4. Demoule A, Jung B, Prodanovic H, Molinari N, Chanques G, Coirault $\mathrm{C}$, et al. Diaphragm dysfunction on admission to the intensive care unit. prevalence, risk factors, and prognostic impact-a prospective study. Am J Respir Crit Care Med 2013;188(2):213-219.

5. Llamas-Álvarez AM, Tenza-Lozano EM, Latour-Pérez J. Diaphragm and lung ultrasound to predict weaning outcome: systematic review and meta-analysis. Chest 2017;152(6):1140-1150.

6. Levy MM, Fink MP, Marshall JC, Abraham E, Angus D, Cook D, et al.; SCCM/ESICM/ACCP/ATS/SIS. 2001 SCCM/ESICM/ACCP/ ATS/SIS International Sepsis Definitions Conference. Crit Care Med 2003;31(4):1250-1256.

7. Khan J, Harrison TB, Rich MM, Moss M. Early development of critical illness myopathy and neuropathy in patients with severe sepsis. Neurology 2006;67(8):1421-1425.

8. Jung B, Nougaret S, Conseil M, Coisel Y, Futier E, Chanques G, Molinari N, et al. Sepsis is associated with a preferential diaphragmatic atrophy: a critically ill patient study using tridimensional computed tomography. Anesthesiology 2014;120(5):1182-1191.

9. Jung B, Moury PH, Mahul M, de Jong A, Galia F, Prades A, et al. Diaphragmatic dysfunction in patients with ICU-acquired weakness and its impact on extubation failure. Intensive Care Med 2016;42(5): 853-861.

10. Dres M, Dubé BP, Mayaux J, Delemazure J, Reuter D, Brochard L, et al. Coexistence and impact of limb muscle and diaphragm weakness at time of liberation from mechanical ventilation in medical intensive care unit patients. Am J Respir Crit Care Med 2017;195(1): 57-66.

11. Blumhof S, Wheeler D, Thomas K, McCool FD, Mora J. Change in diaphragmatic thickness during the respiratory cycle predicts extubation success at various levels of pressure support ventilation. Lung 2016;194(4):519-525.

12. Ferrari G, De Filippi G, Elia F, Panero F, Volpicelli G, Aprà F. Diaphragm ultrasound as a new index of discontinuation from mechanical ventilation. Crit Ultrasound J 2014;6(1):8.

13. Wu A, Drummond GB. Respiratory muscle activity and respiratory obstruction after abdominal surgery. Br J Anaesth 2006;96(4):510515 .

14. Umbrello M, Formenti P, Longhi D, Galimberti A, Piva I, Pezzi A, et al. Diaphragm ultrasound as indicator of respiratory effort in critically ill patients undergoing assisted mechanical ventilation: a pilot clinical study. Crit Care 2015;19:161.

15. Spadaro S, Grasso S, Mauri T, Dalla Corte F, Alvisi V, Ragazzi R, et al. Can diaphragmatic ultrasonography performed during the Ttube trial predict weaning failure? The role of diaphragmatic rapid shallow breathing index. Crit Care; 2016;20(1):305.

16. Yang KL and Tobin MJ. A prospective study of indexes predicting the outcome of trials of weaning from mechanical ventilation. N Engl J Med 1991;324(21):1445-1450. 